\title{
Food Utilization in Artemia for Growth, Reproduction, and Maintenance
}

\author{
Yoshihachiro Nimura, Kenji Nanba, and Md. Idris Miah ${ }^{\dagger}$ \\ Department of Fishery Sciences, University of Tokyo, Bunkyo, Tokyo 113, Japan \\ (Received September 10, 1993)
}

\begin{abstract}
Using marine Chlamydomonas as food, the food requirement of bisexual brine shrimp Artemia franciscana was estimated in terms of nitrogen in order to assist production. The ingested food was theoretically fractionated to the maintenance, somatic growth and reproduction of nauplius and cyst. While feeding marine chlamydomonad ( $5 \mathrm{pg} \mathrm{N} / \mathrm{cell})$ to the shrimp $(L \mathrm{~mm})$, the daily maintenance ration was almost proportional to the cube of body length (2.952) and was 2844 cells/shrimp/day for shrimp of $1 \mathrm{~mm}$ length at $28^{\circ} \mathrm{C}$. On the basis of ingested food, the somatic food conversion efficiency was $67 \%$. The efficiency of nauplius production was $23 \%$ and that of cyst production was $44 \%$, based on the food taken by a pair of shrimps. Including the reproductive products as growth, the gross conversion efficiency was $16 \%$ in the groups from which the maintenance ration and somatic conversion factor were obtained, and $24 \%$ in the groups from which the reproductive conversion factors were obtained. The reproductive growth per total growth increased with the body length and the feeding rate, and was zero below $5.7 \mathrm{~mm}$ in length and 1.0 times the daily maintenance ration.
\end{abstract}

Key words: Artemia, Chlamydomonas, nitrogen budget, growth efficiency, maintenance ration

The worldwide expansion of marine fish-seedling production requires large quantities of brine shrimp Artemia cysts, from which the nauplii are obtained and used as food. Japan imports considerable quantities of the world's cyst production. ${ }^{\left.1{ }^{2}\right)}$ The increasing demand is extending Artemia production to many countries, even to places where the shrimp were not originally found. Therefore, further biological information on Artemia production is in great demand.

The shrimp can consume a large amount of food relative to body mass, but growth is retarded by overfeeding. ${ }^{3)}$ It is important to determine the optimum feeding regime for effective production, especially for intensive culture. Of the many models available to analyze the energy or mass budget, we employed a mass budget model for individual growth including reproduction using nitrogen as the indicator. Nitrogen is one of the important biological elements which constitute the tissue, and the nitrogen content is reported to be 5 to $10 \%$ of the dry weight of shrimp. ${ }^{4)}$ The model used assumes that the assimilated food is used to maintain the body, to increase the body mass and to reproduce offspring such as nauplii and cysts of shrimp.

The present paper describes the regression between the maintenance ration and body length, food conversion factors for somatic growth and reproduction of nauplii and cysts, and the ratio of reproductive growth to total growth. These data are fundamental to know the food requirement of the shrimp.

\section{Materials and Methods}

Marine Chlamydomonas sp. was cultured in Miquel seawater without addition of silicate and used as food in the exponential phase. It contained $5.0 \mathrm{pg} \mathrm{N} / \mathrm{cell}^{3,4)}$ The algal concentration was measured by a Bürker-Türk hemocytometer and the standard error was about $6 \%$. The mean specific growth rate was found to be $0.665 / \mathrm{day}$, which was used to calculate the ration. The stock culture was diluted with $\mathrm{GF} / \mathrm{C}$-filtered seawater to prepare the rearing medium.

The San Francisco Bay brine shrimp Artemia franciscana (previously Artemia salina) was purchased from Metaframe Corporation, Newark, Cal., hatched in seawater at about $28^{\circ} \mathrm{C}$, reared under stable light conditions, fed with the marine chlamydomonad, and kept in the medium at $\mathrm{ca} .28^{\circ} \mathrm{C}$. The body length $L$ was measured under a microscope from the tip of head to the end of furca excluding setae. ${ }^{3)}$ The nitrogen content was estimated from the body length according to the regression for groups including both sexes: ${ }^{4}$ )

$$
\begin{array}{lc}
L, \mathrm{~mm} & \mathrm{~N} \text { content, } \mathrm{ngN} / \mathrm{shrimp} \\
0.8-6.3 & 305.8 L^{2.176} \\
6.3 & 111.7 L^{2.725}
\end{array}
$$

Before starting the feeding experiments, the shrimps were grouped by size and also by sex when they were mature enough for sex identification. A single pair of shrimps of similar size were reared in a beaker to obtain the food conversion factor for reproduction. However, 10 shrimps of similar size were reared in a container to obtain the somatic food conversion factor or daily maintenance ration.

A nauplius was assumed to have $0.1983 \mu \mathrm{g} \mathrm{N} / \mathrm{embryo}$ when spawned and a cyst to have $0.3123 \mu \mathrm{g} \mathrm{N} /$ cyst according to Dutrieu. $^{\text {s) }}$

\section{Estimation of Daily Ration}

The daily ration $d$ was estimated by the following equation: ${ }^{6,7}$

$$
\begin{aligned}
d & =C_{0} \cdot f \cdot[\exp \{(p-f / V) \cdot t\}-1] /(p-f / V), \quad \text { and } t=1 \text { day } \\
& =C_{0} \cdot V \cdot[\exp (p-f / V)-1] /(p \cdot V / f-1) \\
& =C_{0} \cdot V \cdot C f
\end{aligned}
$$

The definition of all the variables including the above are shown in the Appendix in alphabetical order. The correction factor $C f$ shown in Table 1 is nearly unity when $f / V$ is kept large enough. The ratio actually ranged from 2.3 to 21.7 (mainly 3.3-21.2). This phenomenon of $C f$ being almost unity simplified the design of the feeding plan. However, $C f$ was not assumed to be unity in the final calculation.

The filtering rate $f$ was estimated from the body length $L(\mathrm{~mm})$ according to the following regression: ${ }^{31}$

\footnotetext{
+ Present address: Department of Aquaculture and Management, Bangladesh Agricultural University, Mymensingh 2202, Bangladesh
} 
Table 1. Correction factor $(C f)$ to estimate the daily ration by $C_{0} \cdot V$ as related to the change in the filtering rate $(f)$ and the specific phytoplankton growth rate $(p)$

Growth rate
$p, 1 /$ day

Refer Appendix for variables.

${ }^{*} C f=f / V \cdot \exp (p-f / V) /(p-f / V)$.

$$
f=4.989 L^{1.751}, \quad \mathrm{~m} / \text { shrimp/day }
$$

(6)

Mean initial food concentration $C_{0}$ (Tables 2 and 3 ) and mean specific algal growth rate $(p=0.665 /$ day $)$ were used in the calculation. The rearing media including controls without Artemia were changed daily and the body length was measured when the shrimp were transferred to a new container.

Design of Feeding Experiment

The assimilated food is considered to contribute to maintenance of the body, individual or somatic growth and reproduction such as production of nauplii or cysts, as shown by the following equation:

$$
a^{\prime} \cdot R=A=M+g m \cdot G+y n \cdot Y_{n} / 2+y c \cdot Y_{c} / 2
$$

where $a^{\prime}$ is the apparent assimilation efficiency (here $a^{\prime}$ is normally assumed to be unity), $R$ the ration in a certain period $T$ or $T a, M$ the maintenance ration during the period $T, \mathrm{gm}$ the food conversion factor (food per growth) for somatic growth $G, y n$ the factor for the naupliar production $Y_{n}$, and $y_{c}$ the factor for cyst production $Y_{c} . Y_{n}$ and $Y_{c}$ are the spawn per pair as shown in the Appendix.

Estimation of Daily Maintenance Ration

At a fixed daily ration $d l$, shrimps were reared and the body length was measured daily. If growth was confirmed to stop at body length $L l, d l$ was considered to be the daily maintenance ration for the shrimp of body length $L I$ as shown in Fig. 1 . The daily ration was then increased to $d 2$ by decreasing the rearing density, i.e. increasing $V$. When the shrimp attained another steady size $L 2$, another daily maintenance ration was obtained as $d 2$ in the same way. The daily maintenance ration $m t_{\mathrm{i}}$ for each group of shrimps between these sizes was estimated by interpolation using the allometric growth of the group $(\log -\log$ plot). Another kind of daily maintenance ration $m_{\mathrm{r}}$ was obtained from the regression line shown in Fig. 4 (Eqs. (10) and (11)).

\section{Estimation of Somatic Food Conversion Factor}

The simplest way of estimation is described at first, though several kinds of estimation were carried out. As shown in Fig. 1, the daily maintenance ration $m_{\mathrm{i}}$ for a group is estimated from the body length growth curve. The integrated difference $\left(d 2-m_{\mathrm{i}}\right)$ over period $T$ is considered to be the surplus food for the somatic or individual growth before the shrimp starts to reproduce, and is shown by the shaded area in Fig. 1. Assuming that $a^{\prime}$ is unity, the ratio of all the surplus food $\left(R m_{\mathrm{i}}=A-M_{\mathrm{i}}\right.$ in Table 2) to the somatic growth $G$ is defined here as the somatic food conversion factor $g m_{\mathrm{i}}$ in terms of nitrogen, i.e., $g m_{\mathrm{i}}=R m_{\mathrm{i}} / G$ in Table 2. This mean is $g m_{\mathrm{i} 1}$ in Table 4 ( $c f$. Appendix for details of the variables).

The second estimation method used the ratio $g m_{\mathrm{r}}$ for each group, $M_{\mathrm{r}}$, instead of $M_{\mathrm{i}}$

$$
g m_{\mathrm{r}}=\left(A-M_{\mathrm{r}}\right) / G=R m_{\mathrm{r}} / G
$$

as shown in Table 2. This mean is $g m_{r 1}$ in Table 4

The third and fourth estimates of $g m$ were the weighted means of $g m_{i}$

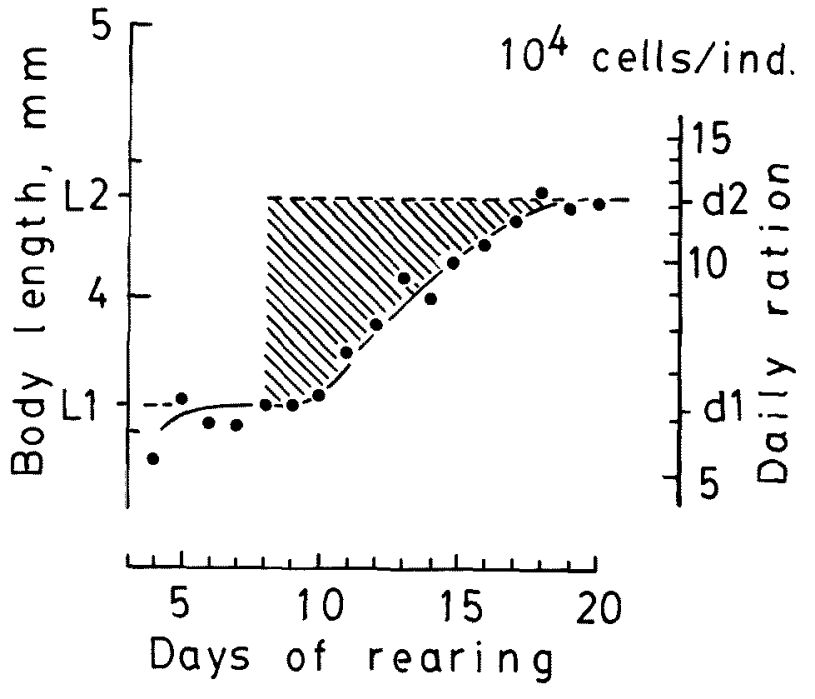

Fig. 1. Experimental design to estimate the daily maintenance ration and food conversion factor in Artemia.

Solid circles are the body length of shrimp and the solid line is the growth curve. Broken lines are the fixed daily rations and the shaded area is the ration which contributes to the growth of shrimp. $L l$ and $L 2$ are the steady length attained at the daily ration of $d I$ and $d 2$, respectively.

and $g m_{r}$, respectively, $i_{.}$, , the ratios of mean ration over maintenance to mean somatic growth. These are $g m_{\mathrm{i} 2}$ and $g m_{\mathrm{r} 2}$ in Table 4.

The fifth and sixth estimations were made by multiple regression using data $R m_{\mathrm{i}}$ and $R m_{\mathrm{r}}$ including the groups which reproduced. These are $g m_{\mathrm{ir}}$ and $g m_{r}$ in Table 4.

The seventh estimate of $g m_{3}$ used to estimate the reproductive food conversion factor was obtained from the regression of $g m_{\mathrm{i}} v s$. body length $L$ as shown in Fig. 5 assuming that the factor changed with body size.

These factors are shown below (cf. Appendix):

$g m_{\mathrm{i} 1}=\operatorname{Mean}\left(g m_{\mathrm{i}}\right), g m_{\mathrm{i}}=R m_{\mathrm{i}} / G=\left(A-M_{\mathrm{i}}\right) / G$

$g m_{\mathrm{r} 1}=\operatorname{Mean}\left(g m_{\mathrm{r}}\right), g m_{\mathrm{r}}=R m_{\mathrm{r}} / G=\left(A-M_{\mathrm{r}}\right) / G$;

$g m_{\mathrm{i} 2}=\Sigma R m_{\mathrm{i}} / \Sigma G$

$g m_{\mathrm{r} 2}=\Sigma R m_{\mathrm{r}} / \Sigma G$

$g m_{\mathrm{ir}}$ by multiple regression of $R m_{\mathrm{i}}\left(G, Y_{c}, Y_{n}\right)$ and $R m_{\mathrm{r}}\left(G, Y_{c}, Y_{n}\right)$;

$g m_{\mathrm{rr}}$ by multiple regression of $R m_{\mathrm{r}}\left(G, Y_{c}, Y_{n}\right)$; and

$g m_{3}$ by regression of $g m_{i}(L)$ 
Estimation of Reproductive Food Conversion Factor

A fixed daily ration was given to each pair of shrimps throughout the experiment. However, the ration differed among the pairs. $R y_{\mathrm{x}}$, the tation over maintenance and somatic growth, is considered to contribute to the production of nauplii $Y n$ and/or cysts $Y c: A-M-a m_{x} \cdot G$ where the suffix $x$ denotes the estimation method as shown in Table 4 and the Appendix. The component of $R y_{\mathrm{x}}$ for $Y n_{\mathrm{x}}$ divided by $Y n_{\mathrm{x}}$ is defined to be the reproductive food conversion factor $y n_{x}$, and $y c_{x}$ is also defined in the same way. These were estimated by the multiple regression described in the next section (Statistical analysis). However, there are many ways of estimating $M$ and $g m_{\mathrm{x}}$ as shown above. Using the ration over maintenance $R m_{i}$ is a compromise when using methods ir, ril, or ri2 $(\mathrm{cf}$. Table 4 and the end of the above section), i.e., $R m_{i}$ for groups $1-17$ and $R m_{\mathrm{r}}$ for groups 18-35. The estimate $\mathrm{gm}_{3}$ was also used in the same way, in which only $R y_{r}$ in Table 3 was used as the dependent variable.

Another problem is the choice of period $T a$ during which the ration directly contributes to the production of nauplii and/or cysts. Shrimp usually spawn every 3 days in the optimum conditions at the temperature. This interval is the reproductive cycle. The oocytes pass out from the ovary to the brood pouch (ovisac) 2 or 3 days before being spawned and are inseminated. The embryos develop there into nauplii or cysts which are covered by the shell secreted by shell glands in the inner wall of the brood pouch. Assuming that the materials for reproduction are accumulated over longer period than the reproductive cycle ( 2 or 3 days per cycle) and that the brood size (number of young produced per brood) is stable, then the surplus ration for reproduction per brood is equal to the increase in the surplus in a cycle except the initial cycle as shown in Fig. 2. In this figure, $r y$ is the maximum food surplus for reproduction per brood and $n$ is the number of reproductive cycles in accumulating materials for a brood:

$$
r y=r y / n \cdot\left(\sum_{j=1}^{n} j-\sum_{j=1}^{n-1} j\right)=r y / n \cdot n
$$

Statistical Analysis

Letting the surplus food for somatic growth and/or reproduction be the

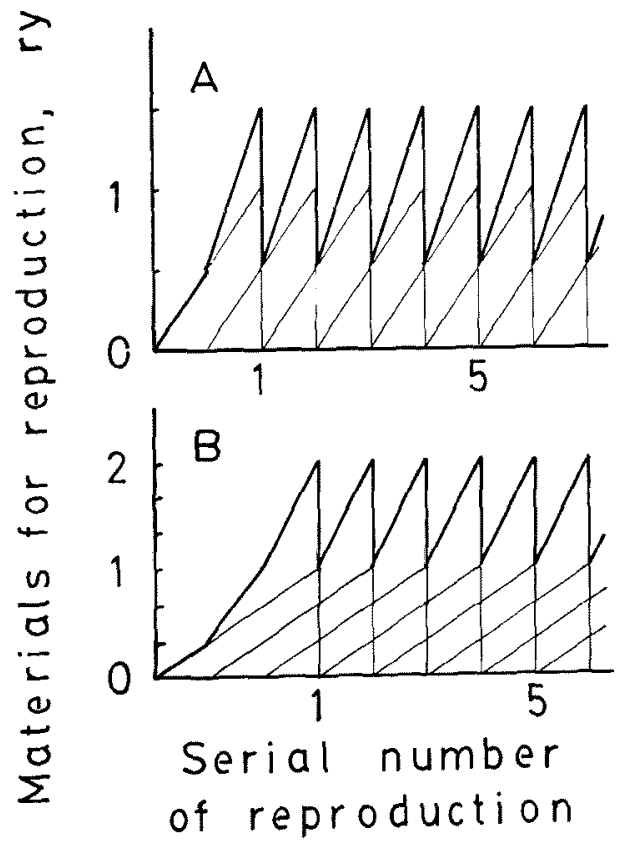

Fig. 2. Schematic diagram of estimating the ration which contributes to reproduction

Bold lines show the sum of materials which are accumulated and metabolized for reproduction, and thin lines show those for each spawning or reproduction. Figures $2 \mathrm{~A}$ and $2 \mathrm{~B}$ show the case where the materials for reproduction are accumulated and metabolized over two reproductive cycles $(n=2)$ and over three cycles $(n=3)$, respectively. Materials for reproduction are shown in units of $r y$ (material for reproduction per brood per pair). For further details, see the text. dependent variable and $G, Y c$, and $Y n$ be the independent variables, multiple regression was applied to the partly normalized data in order not to degrade the accuracy of the matrix calculation. ${ }^{8)}$ The regression equation was assumed to pass through the origin. ${ }^{91}$ However, the log-log regression between the daily maintenance ration and body length was not assumed to pass through the origin. The regression equation (12) was obtained by eye at first to determine the asymptote with the help of the coefficient of determination $\left(R^{2}\right)$. Equation (13) was also obtained in the same way.

The standard errors (SE) of the ratios, $g m_{i 2}$ and $g m_{r 2}$, were calculated according to the formula given by Kuno. ${ }^{10)}$

\section{Results}

Daily Maintenance Ration vs. Body Length

Figure 3A shows examples of the growth curves with the first and second cessations of growth. Some of the data are also shown in Table 2 . In several cases, however, only the initial cessation was observed and those data were also used for the analysis.

A straight line was fitted to the log-log plot of the daily maintenance ration $m L$ and body length $L$ as shown in Fig. 4:

$$
m_{\mathbf{r}}=2844 \cdot L^{2.952} \quad(N=61) \quad \text { cells/shrimp/day }
$$

and

$$
=14.22 \cdot L^{2.952} \quad \mathrm{ng} \mathrm{N} / \mathrm{shrimp} / \text { day } .
$$

The standard error of the exponent 2.952 was 0.1373 and that of $\log _{10} 2844$ was 0.0851 .

The logarithmic slope for each group Slpi ranged from 2.13 to 4.14 , and the mean was 3.015 as shown in Table 2 .

\section{Somatic Food Conversion Factor}

Table 2 shows the data of non-spawners which ceased growth two times in the rearing experiment. There were 17 groups. The largest mean body length was $6.23 \mathrm{~mm}$. The surplus food for growth was estimated as $R m_{\mathrm{i}}$ or $R m_{\mathrm{r}}$ for each group. The factor $g m_{i}\left(=R m_{i} / G\right)$ ranged from 0.40 to 3.07 and the mean was $1.292\left(=g m_{\mathrm{i} 1}\right.$ in Table 4$)$, while the weighted mean was $1.489\left(=g m_{\mathrm{i} 2}\right.$ in Table 4$)$ which was the ratio of the sum of $R m_{\mathrm{i}}$ to the sum of $G$. The factor $g m_{\mathrm{i}}$ increased with body size (Fig. $5, r \neq 0, p<0.01$ ). The regression was obtained as follows:

$$
\log _{10}\left(R m_{\mathrm{i}} / G-0.384\right)=-16.266 / L^{2}+0.798,
$$

where $L$ is the body length in $\mathrm{mm}$. The coefficient of determination due to regression was 0.777 . The estimates from the regression are shown as $g m_{3}$ in Tables 2,3 , and 4. However, the distribution of the square root of this ratio $\left(R m_{\mathrm{i}} / G\right)$ was almost symmetric.

As shown in Table 2, the factor $g m_{\mathrm{r}}\left(=R m_{\mathrm{r}} / G\right)$ ranged from -2.12 to 4.29 and the mean was $1.520\left(=g m_{\mathrm{r} 1}\right.$ in Table 4$)$, while the weighted mean was $2.095\left(=g m_{\mathrm{r} 2}\right.$ in Table 4 ). The above factors were obtained only by using the data of non-spawners which did not reproduce during the experiment.

Two other factors were estimated from the multiple regression using all the data including data for groups which reproduced: $R m_{\mathrm{i}}$ and/or $R m_{\mathrm{r}}$ was the dependent variable and $G, Y c$, and $Y n$ were the independent variables. These are $g m_{\mathrm{ir}}$ and $g m_{\mathrm{rr}}$ in Table 4, i.e., $g m_{\mathrm{ir}}=1.122$ and $g m_{\mathrm{rr}}=$ 1.833 .

The gross conversion factor was found to be 6.1 (= $16.4 \%$ ) for groups $1-17$ as shown at the bottom of column 
Table 2. Feeding and growth of Artemia which did not reproduce in the rearing experiment to estimate the somatic food conversion factor

\begin{tabular}{|c|c|c|c|c|c|c|c|c|c|c|c|c|c|c|c|c|c|c|c|c|c|c|c|}
\hline \multirow[t]{2}{*}{ Group } & \multirow[t]{2}{*}{$C_{0}$} & \multirow[t]{2}{*}{$\begin{array}{c}V \\
\mathrm{~m} l\end{array}$} & \multirow[t]{2}{*}{$\begin{array}{c}T \\
\text { day }\end{array}$} & \multirow[t]{2}{*}{$\begin{array}{c}L l \\
\mathrm{~mm}\end{array}$} & \multirow[t]{2}{*}{$\begin{array}{c}L 2 \\
\mathrm{~mm}\end{array}$} & \multirow[t]{2}{*}{$\underset{\mathrm{m} l}{f}$} & \multirow[t]{2}{*}{$d$} & \multirow[t]{2}{*}{$\begin{array}{c}A \\
\mu \mathrm{g} \mathrm{N}\end{array}$} & \multirow[t]{2}{*}{$\begin{array}{c}M_{\mathrm{i}} \\
\mu \mathrm{g} \mathrm{N}\end{array}$} & \multirow[t]{2}{*}{$\begin{array}{c}M_{\mathrm{r}} \\
\mu \mathrm{g} \mathrm{N}\end{array}$} & \multirow[t]{2}{*}{$\begin{array}{c}G \\
\mu \mathrm{g} \mathrm{N}\end{array}$} & \multirow[t]{2}{*}{$S l p i$} & \multirow[t]{2}{*}{$\begin{array}{l}R m_{\mathrm{j}} \\
\mu \mathrm{g} \mathrm{N}\end{array}$} & \multirow[t]{2}{*}{$\begin{array}{l}R m_{\mathrm{r}} \\
\mu \mathrm{g} \mathrm{N}\end{array}$} & \multirow[t]{2}{*}{$g m_{i}$} & \multirow[t]{2}{*}{$g m_{x}$} & \multirow[t]{2}{*}{$g m_{3}$} & \multirow{2}{*}{$\begin{array}{r}R y_{\mathrm{i} 1} \\
\mu \mathrm{g} \mathrm{N}\end{array}$} & \multirow{2}{*}{$\begin{array}{l}R y_{\mathrm{i} 2} \\
\mu \mathrm{g} \mathrm{N} \\
\text { Used so } \\
1.489\end{array}$} & \multirow{2}{*}{$\begin{array}{c}R y_{13} \\
\mu \mathrm{g} \mathrm{N} \\
\text { matic co }\end{array}$} & \multirow{2}{*}{$\begin{array}{c}\begin{array}{c}R y_{\mathrm{r} 1} \\
\mu \mathrm{g} \mathrm{N}\end{array} \\
\text { onversion }\end{array}$} & $\begin{array}{r}R y_{\mathrm{r} 2} \\
\mu \mathrm{g} \mathrm{N} \\
\text { factor }\end{array}$ & \multirow{2}{*}{$\begin{array}{c}R y_{\mathrm{r}} 3 \\
\mu \mathrm{g} \mathrm{N} \\
g m_{3}\end{array}$} \\
\hline & & & & & & & & & & & & & & & & & & & & & & 2.095 & \\
\hline \multirow[t]{2}{*}{1} & 83.7 & 2 & 17 & 3.18 & & 38 & 174 & 38.4 & 28.8 & 18.5 & 5.9 & 2.125 & 9.6 & 20.0 & 1.621 & 3.371 & 1.018 & 2.0 & 0.8 & 3.6 & 11.0 & 7.6 & 13.9 \\
\hline & $\begin{array}{l}83.7 \\
83.7\end{array}$ & $\begin{array}{l}5 \\
2\end{array}$ & 16 & & 4.90 & 81 & 436 & & & & & & & & & & & & & & & & \\
\hline 2 & $\begin{array}{l}83.7 \\
83.7\end{array}$ & 5 & 16 & 3.20 & 4.87 & $\begin{array}{l}38 \\
80\end{array}$ & $\begin{array}{l}174 \\
441\end{array}$ & 36.2 & 28.4 & 18.2 & 5.7 & 2.213 & 7.8 & 18.0 & 1.352 & 3.140 & 1.014 & 0.3 & -0.8 & 1.9 & 9.3 & 6.0 & 12.2 \\
\hline 3 & $\begin{array}{l}83.7 \\
83.7\end{array}$ & $\begin{array}{l}2 \\
5\end{array}$ & 17 & 3.39 & 4.90 & $\begin{array}{l}42 \\
81\end{array}$ & $\begin{array}{l}174 \\
436\end{array}$ & 38.4 & 28.3 & 19.1 & 5.4 & 2.504 & 10.1 & 19.3 & 1.892 & 3.608 & 1.095 & 3.2 & 2.2 & 4.3 & 11.2 & 8.1 & 13.5 \\
\hline 4 & $\begin{array}{l}83.7 \\
83.7\end{array}$ & $\begin{array}{l}2 \\
5\end{array}$ & 18 & 3.32 & 4.95 & $\begin{array}{l}41 \\
82\end{array}$ & $\begin{array}{l}174 \\
438\end{array}$ & 40.6 & 30.1 & 20.3 & 5.8 & 2.317 & 10.5 & 20.3 & 1.828 & 3.522 & 1.087 & 3.1 & 2.0 & 4.3 & 11.5 & 8.2 & 14.0 \\
\hline 5 & $\begin{array}{l}33.5 \\
33.5\end{array}$ & $\begin{array}{l}2 \\
5\end{array}$ & 12 & 2.75 & 4.00 & $\begin{array}{l}29 \\
57\end{array}$ & $\begin{array}{r}70 \\
194\end{array}$ & 11.3 & 8.9 & 7.6 & 3.5 & 2.707 & 2.3 & 3.6 & 0.6662 & 1.041 & 0.6186 & -2.2 & -2.9 & 0.2 & -1.7 & -3.7 & 1.5 \\
\hline 6 & $\begin{array}{l}33.5 \\
33.5\end{array}$ & $\begin{array}{l}2 \\
5\end{array}$ & 11 & 2.50 & 3.80 & $\begin{array}{l}25 \\
52\end{array}$ & $\begin{array}{r}71 \\
180\end{array}$ & 10.3 & 8.2 & 6.2 & 3.3 & 2.216 & 2.2 & 4.2 & 0.6472 & 1.243 & 0.5282 & -2.2 & -2.8 & 0.4 & -0.9 & -2.8 & 2.4 \\
\hline 7 & $\begin{array}{l}33.5 \\
33.5\end{array}$ & $\begin{array}{l}10 \\
20\end{array}$ & 14 & 5.08 & 6.10 & $\begin{array}{r}86 \\
118\end{array}$ & $\begin{array}{l}366 \\
781\end{array}$ & 55.2 & 39.5 & 33.2 & 5.1 & 4.140 & 15.8 & 22.0 & 3.068 & 4.285 & 2.280 & 9.1 & 8.1 & 4.0 & 14.2 & 11.3 & 10.3 \\
\hline 8 & $\begin{array}{l}33.5 \\
33.5\end{array}$ & $\begin{array}{l}10 \\
20\end{array}$ & 14 & 4.80 & 6.00 & $\begin{array}{r}78 \\
115\end{array}$ & $\begin{array}{l}369 \\
783\end{array}$ & 55.4 & 41.6 & 31.1 & 5.8 & 3.369 & 13.8 & 24.3 & 2.375 & 4.181 & 2.124 & 6.3 & 5.1 & 1.5 & 15.4 & 12.1 & 11.9 \\
\hline 9 & $\begin{array}{l}33.5 \\
33.5\end{array}$ & $\begin{array}{l}10 \\
20\end{array}$ & 14 & 5.06 & 6.23 & $\begin{array}{r}85 \\
123\end{array}$ & $\begin{array}{l}367 \\
779\end{array}$ & 55.0 & 42.3 & 36.2 & 6.0 & 3.623 & 12.6 & 18.8 & 2.121 & 3.159 & 2.324 & 4.9 & 3.8 & -1.2 & 9.8 & 6.3 & 5.0 \\
\hline 10 & $\begin{array}{l}13.4 \\
13.4\end{array}$ & $\begin{array}{l}2 \\
5\end{array}$ & 14 & 2.18 & 3.20 & $\begin{array}{l}20 \\
38\end{array}$ & $\begin{array}{l}29 \\
77\end{array}$ & 5.3 & 4.5 & 4.9 & 2.2 & 2.558 & 0.9 & 0.4 & 0.4048 & 0.1859 & 0.4195 & -1.9 & -2.4 & 0.0 & -2.9 & -4.2 & -0.5 \\
\hline 11 & $\begin{array}{l}13.4 \\
13.4\end{array}$ & $\begin{array}{l}2 \\
5\end{array}$ & 16 & 2.25 & 3.46 & $\begin{array}{l}21 \\
44\end{array}$ & $\begin{array}{l}29 \\
73\end{array}$ & 6.0 & 4.7 & 6.6 & 2.8 & 2.171 & 1.3 & -0.6 & 0.4714 & -0.2054 & 0.4475 & -2.3 & -2.8 & 0.1 & -4.8 & -6.4 & -1.8 \\
\hline 12 & $\begin{array}{l}13.4 \\
13.4\end{array}$ & $\begin{array}{l}10 \\
20\end{array}$ & 12 & 4.07 & 5.00 & $\begin{array}{l}58 \\
84\end{array}$ & $\begin{array}{l}152 \\
315\end{array}$ & 19.4 & 14.9 & 16.3 & 3.7 & 3.543 & 4.5 & 3.1 & 1.226 & 0.8354 & 1.401 & -0.2 & -1.0 & -0.6 & -2.5 & -4.6 & -2.1 \\
\hline 13 & $\begin{array}{l}13.4 \\
13.4\end{array}$ & $\begin{array}{l}10 \\
20\end{array}$ & 14 & 3.95 & 5.05 & $\begin{array}{l}55 \\
85\end{array}$ & $\begin{array}{l}153 \\
321\end{array}$ & 22.5 & 17.9 & 19.0 & 4.3 & 3.022 & 4.6 & 3.5 & 1.074 & 0.8117 & 1.373 & -0.9 & -1.8 & -1.3 & -3.0 & -5.5 & -2.4 \\
\hline 14 & $\begin{array}{l}13.4 \\
13.4\end{array}$ & $\begin{array}{l}10 \\
20\end{array}$ & 12 & 4.07 & 4.95 & $\begin{array}{l}58 \\
82\end{array}$ & $\begin{array}{l}152 \\
315\end{array}$ & 19.4 & 15.2 & 16.3 & 3.4 & 3.729 & 4.2 & 3.1 & 1.207 & 0.8901 & 1.381 & -0.3 & -1.0 & -0.6 & -2.2 & -4.1 & -1.7 \\
\hline 15 & $\begin{array}{l}5.4 \\
5.4\end{array}$ & $\begin{array}{l}10 \\
20\end{array}$ & 10 & 3.55 & 4.35 & $\begin{array}{l}46 \\
65\end{array}$ & $\begin{array}{r}62 \\
131\end{array}$ & 6.5 & 5.1 & 9.0 & 2.7 & 3.680 & 1.4 & -2.6 & 0.5122 & -0.9534 & 0.9539 & -2.1 & -2.6 & -1.2 & -6.6 & -8.2 & -5.1 \\
\hline 16 & $\begin{array}{l}5.4 \\
5.4\end{array}$ & $\begin{array}{l}10 \\
20\end{array}$ & 11 & 3.60 & 4.35 & $\begin{array}{l}47 \\
65\end{array}$ & $\begin{array}{r}62 \\
123\end{array}$ & 7.1 & 5.3 & 10.0 & 2.5 & 3.610 & 1.8 & -2.9 & 0.6973 & -1.152 & 0.9713 & -1.5 & -2.0 & -0.7 & -6.8 & -8.2 & -5.4 \\
\hline 17 & $\begin{array}{l}5.4 \\
5.4\end{array}$ & $\begin{array}{l}10 \\
20\end{array}$ & 13 & 3.88 & 4.65 & $\begin{array}{l}54 \\
74\end{array}$ & $\begin{array}{r}61 \\
120\end{array}$ & 8.4 & 6.1 & 14.4 & 2.8 & 3.724 & 2.3 & -6.0 & 0.8012 & -2.122 & 1.186 & -1.4 & -1.9 & -1.1 & -10.3 & -11.9 & -9.3 \\
\hline Mean & 34.4 & 9.6 & 13.8 & 3.58 & 4.75 & 63 & 252 & 25.6 & 19.4 & 16.9 & 4.2 & 3.015 & 6.2 & 8.7 & 1.292 & 1.520 & 1.189 & 0.8 & 0.0 & 0.8 & 2.4 & 0.0 & 3.3 \\
\hline $\begin{array}{l}\text { SE } \\
\text { Food/G }\end{array}$ & $\begin{array}{r}5.1 \\
\text { owth }\end{array}$ & 1.2 & 0.6 & 0.22 & 0.21 & 5 & 36 & $\begin{array}{c}4.5 \\
6.143\end{array}$ & 3.4 & 2.3 & 0.3 & 0.168 & $\begin{array}{c}1.2 \\
1.489\end{array}$ & $\begin{array}{c}2.5 \\
2.095\end{array}$ & $\begin{array}{l}0.185 \\
1.292\end{array}$ & $\begin{array}{l}0.488 \\
1.520\end{array}$ & $\begin{array}{c}0.143 \\
\text { var. }\end{array}$ & 0.8 & 0.8 & 0.5 & 2.1 & 1.9 & 1.9 \\
\hline
\end{tabular}

Refer Appendix for variables. 
Table 3. Feeding, growth, and reproduction of Artemia which reproduced in the rearing experiment to estimate the reproductive food conversion factors

\begin{tabular}{|c|c|c|c|c|c|c|c|c|c|c|}
\hline Group & $C_{0}$ & $\begin{array}{c}V \\
\mathrm{~m} l\end{array}$ & $\begin{array}{c}T a \\
\text { day }\end{array}$ & $\begin{array}{l}\text { Lla } \\
\mathrm{mm}\end{array}$ & $\begin{array}{l}L 2 a \\
\mathrm{~mm}\end{array}$ & $\begin{array}{c}f \\
\mathrm{~m} l\end{array}$ & $d$ & $\begin{array}{c}R \\
\mu \mathrm{g} \mathrm{N}\end{array}$ & $\begin{array}{c}M_{\mathrm{s}} \\
\mu \mathrm{g} \mathrm{N}\end{array}$ & $\begin{array}{c}G \\
\mu \mathrm{g} \mathrm{N}\end{array}$ \\
\hline 18 & 83.7 & 60 & 13 & 6.95 & 7.70 & 163 & 5800 & 377.0 & 68.3 & 7.1 \\
\hline 19 & 83.7 & 60 & 10 & 8.00 & 8.50 & 201 & 5837 & 291.9 & 69.4 & 5.8 \\
\hline 20 & 83.7 & 80 & 6 & 7.76 & 8.60 & 198 & 7670 & 230.1 & 43.8 & 9.6 \\
\hline 21 & 83.7 & 20 & 10 & 6.60 & 7.10 & 145 & 1842 & 92.1 & 41.4 & 4.2 \\
\hline 22 & 83.7 & 40 & 3 & 6.54 & 6.80 & 138 & 3891 & 58.4 & 11.5 & 2.1 \\
\hline 23 & 83.7 & 20 & 16 & 6.11 & 7.05 & 135 & 1851 & 148.1 & 60.5 & 7.2 \\
\hline 24 & 83.7 & 40 & 13 & 6.90 & 7.68 & 162 & 3866 & 251.3 & 67.2 & 7.3 \\
\hline 25 & 83.7 & 20 & 17 & 6.77 & 7.30 & 152 & 1830 & 155.5 & 79.2 & 4.7 \\
\hline 26 & 83.7 & 20 & 3 & 6.57 & 7.00 & 143 & 1843 & 27.6 & 12.2 & 3.6 \\
\hline 27 & 83.7 & 80 & 3 & 6.63 & 7.06 & 145 & 7231 & 108.5 & 12.7 & 3.6 \\
\hline 28 & 83.7 & 80 & 5 & 7.54 & 8.08 & 182 & 7570 & 189.2 & 30.7 & 5.7 \\
\hline 29 & 83.7 & 10 & 13 & 6.50 & 6.50 & 132 & 881 & 57.3 & 46.4 & 0.0 \\
\hline 30 & 83.7 & 20 & 6 & 6.87 & 7.28 & 153 & 1830 & 54.9 & 27.9 & 3.6 \\
\hline 31 & 83.7 & 80 & 6 & 7.30 & 7.30 & 162 & 7412 & 222.4 & 30.2 & 0.0 \\
\hline 32 & 83.7 & 10 & 6 & 5.82 & 6.00 & 112 & 889 & 26.7 & 16.6 & 1.0 \\
\hline 33 & 83.7 & 60 & 11 & 7.10 & 7.40 & 160 & 5796 & 318.8 & 56.1 & 2.8 \\
\hline 34 & 83.7 & 40 & 10 & 6.95 & 7.54 & 160 & 3877 & 193.9 & 47.1 & 5.5 \\
\hline 35 & 83.7 & 40 & 14 & 6.48 & 7.11 & 143 & 3885 & 272.0 & 60.1 & 5.2 \\
\hline Mean & 83.7 & 43 & 9.2 & 6.86 & 7.33 & 155 & 4100 & 170.9 & 43.4 & 4.4 \\
\hline $\mathrm{SE}$ & & 6 & 1.1 & 0.13 & 0.15 & 5 & 575 & 25.2 & 5.2 & 0.6 \\
\hline \multirow[t]{3}{*}{$\begin{array}{c}\text { Group } \\
\quad \#\end{array}$} & $\begin{array}{c}Y n \\
\mu \mathrm{g} \mathrm{N}\end{array}$ & $\begin{array}{c}Y_{C} \\
\mu \mathrm{g} \mathrm{N}\end{array}$ & $\begin{array}{l}R m_{\mathrm{r}} \\
\mu \mathrm{g} \mathrm{N}\end{array}$ & $g m_{3}$ & $\begin{array}{l}R y_{\mathrm{r} 1} \\
\mu \mathrm{g} \mathrm{N}\end{array}$ & $\begin{array}{l}R y_{\mathrm{r} 2} \\
\mu \mathrm{gN}\end{array}$ & $\begin{array}{l}R y_{\mathrm{r} 3} \\
\mu \mathrm{g} \mathrm{N}\end{array}$ & $\begin{array}{l}R y_{\mathrm{ril}} \\
\mu \mathrm{g} \mathrm{N}\end{array}$ & $\begin{array}{l}R y_{\mathrm{ri} 2} \\
\mu \mathrm{g} \mathrm{N}\end{array}$ & $\begin{array}{l}R y_{\mathrm{ri} 2 \mathrm{a}} \\
\mu \mathrm{g} \mathrm{N}\end{array}$ \\
\hline & & & & & \multicolumn{6}{|c|}{ Used somatic conversion factor } \\
\hline & & & & & 1.520 & 2.095 & $g m_{3}$ & 1.292 & 1.489 & 1.489 \\
\hline 18 & 0.0 & 247.8 & 308.7 & 3.511 & 298.0 & 293.9 & 283.9 & 299.6 & 298.2 & 298.2 \\
\hline 19 & 0.0 & 195.7 & 222.5 & 4.009 & 213.7 & 210.3 & 199.2 & 215.0 & 213.8 & 213.8 \\
\hline 20 & 0.0 & 135.5 & 186.3 & 3.975 & 171.7 & 166.1 & 148.1 & 173.0 & 172.0 & 172.0 \\
\hline 21 & 0.0 & 63.7 & 50.7 & 3.213 & 44.3 & 41.9 & 37.2 & 45.2 & 44.4 & 44.4 \\
\hline 22 & 0.0 & 35.6 & 46.9 & 3.092 & 43.7 & 42.5 & 40.4 & 44.2 & 43.7 & 43.7 \\
\hline 23 & 34.1 & 39.6 & 87.6 & 3.030 & 76.7 & 72.6 & 65.9 & 78.4 & 77.0 & 77.0 \\
\hline 24 & 59.9 & 62.4 & 184.1 & 3.491 & 173.0 & 168.8 & 158.6 & 174.6 & 173.2 & 173.2 \\
\hline 25 & 35.5 & 12.2 & 76.3 & 3.333 & 69.2 & 66.5 & 60.7 & 70.3 & 69.3 & 69.3 \\
\hline 26 & 12.5 & 0.0 & 15.4 & 3.170 & 10.0 & 8.0 & 4.1 & 10.8 & 10.1 & 10.1 \\
\hline 27 & 26.2 & 0.0 & 95.8 & 3.210 & 90.3 & 88.2 & 84.2 & 91.1 & 90.4 & $55.7^{*}$ \\
\hline 28 & 33.3 & 0.0 & 158.6 & 3.786 & 149.9 & 146.6 & 137.0 & 151.2 & 150.1 & $137.7^{*}$ \\
\hline 29 & 4.0 & 0.0 & 10.9 & 2.974 & 10.9 & 10.9 & 10.9 & 10.9 & 10.9 & 10.9 \\
\hline 30 & 6.5 & 0.0 & 27.0 & 3.358 & 21.5 & 19.4 & 14.8 & 22.3 & 21.6 & 21.6 \\
\hline 31 & 41.2 & 0.0 & 192.2 & 3.497 & 192.2 & 192.2 & 192.2 & 192.2 & 192.2 & $147.0^{*}$ \\
\hline 32 & 3.2 & 0.0 & 10.1 & 2.535 & 8.6 & 8.1 & 7.7 & 8.9 & 8.7 & 8.7 \\
\hline 33 & 64.4 & 0.0 & 262.7 & 3.466 & 258.5 & 256.9 & 253.0 & 259.1 & 258.5 & 258.5 \\
\hline 34 & 92.6 & 0.0 & 146.8 & 3.463 & 138.4 & 135.3 & 127.8 & 139.7 & 138.6 & 138.6 \\
\hline 35 & 113.2 & 0.0 & 211.9 & 3.177 & 203.9 & 200.9 & 195.3 & 205.1 & 204.1 & 204.1 \\
\hline Mean & 29.3 & 44.0 & 127.5 & 3.350 & 120.8 & 118.3 & 112.3 & 121.8 & 120.9 & 115.8 \\
\hline SE & 8.1 & 17.6 & 21.9 & 0.084 & 21.5 & 21.4 & 20.9 & 21.6 & 21.5 & 21.4 \\
\hline
\end{tabular}

Refer Appendix for variables.

* The correction due to the assimilation efficiency was made on these groups as shown in Discussion (p. 50/R), since they ingested a very large amount of food.

$A$ in Table 2. The factor for groups $18-35$ (Table 3 ) was $4.2(=24.0 \%)$ including the spawn as growth.

\section{Reproductive Food Conversion Factors}

There are two kinds of factors, i.e., a factor for nauplius production ( $y n_{\mathrm{x}}$ in Table 4 ) and a factor for cyst production $\left(y c_{x}\right.$ in Table 4). Table 3 shows the data on the groups which spawned in the experiment. There were 18 groups. The smallest mean body length was $5.82 \mathrm{~mm}$ when the initial spawning was observed. The surplus food for reproduction was estimated as $R y_{\mathrm{x}}$ shown in Table 3 , while the suffix $\mathrm{x}$ was ri1, ri2, ri2a, or others. However, these were very similar to each other despite the difference in $g m_{x}$, probably because the somatic growth in the rearing experiment was small $(12 \%)$ compared to the reproduction. The factors were obtained by multiple regression, using the surplus food only for reproduction $\left(R y_{\mathrm{x}}\right)$ and also for all the growth including reproduction $\left(R m_{\mathrm{r}}\right)$. These are shown in the upper part of Table 4 , and the mean (except r3) of $y n_{\mathrm{x}}$ was $4.383(=$ $22.8 \%)$ and that of $y c_{x}$ was $2.258(=44.3 \%)$.

Groups 18-22 spawned only cysts. The ratio of weighted mean of $R y_{x}$ to that of $Y C$ was obtained as $y c_{\mathrm{x}}(\simeq 2.3)$ 


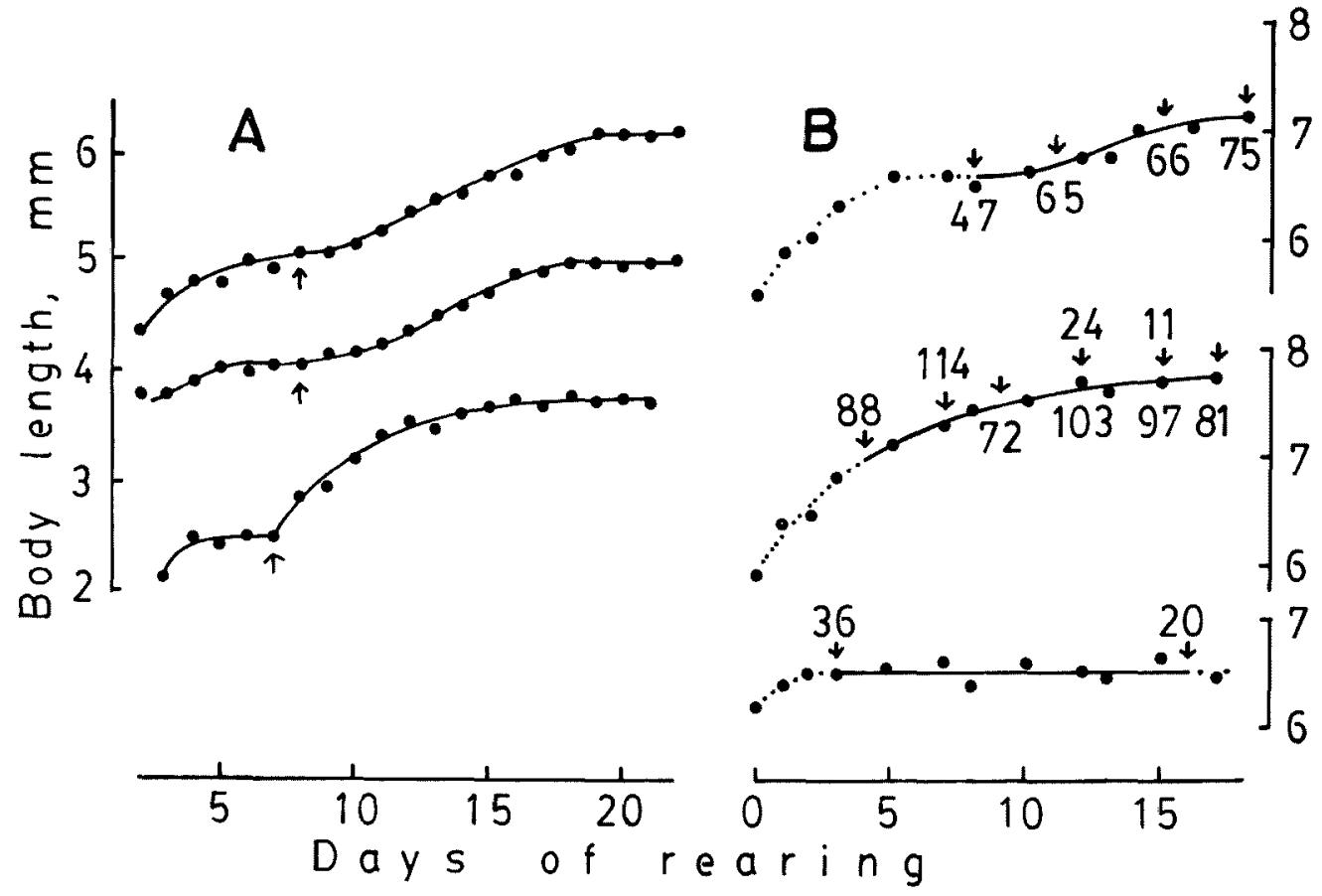

Fig. 3. Examples of growth curves of Artemia franciscana in the experiment.

Figure $3 \mathrm{~A}$ is for groups 9,12 , and 1 from the top. Figure $3 \mathrm{~B}$ is for groups 21,24 , and 29 from the top. Solid circles are observed body length, lines are growth curves fitted by eye, and solid circles in Fig. 3B are used to estimate the ration which contributed to reproduction. Arrows in Fig. $3 \mathrm{~A}$ show the time when the ration was changed, and those in Fig. 3B the time of reproduction observed. The numerals below the lines (Fig. 3B) are the number of cysts produced and those above the lines are the number of nauplii produced.

Table 4. Comparison of food coversion factors estimated by various methods (x)

\begin{tabular}{|c|c|c|c|c|c|c|}
\hline \multicolumn{2}{|c|}{ Based data } & \multicolumn{3}{|c|}{ Food conversion factors for } & \multirow[b]{2}{*}{$\begin{array}{l}\text { Ratio } \\
y n / y c\end{array}$} & \multirow{2}{*}{$\begin{array}{c}\text { Coefficient } \\
\text { of } \\
\text { determination } \\
\mathrm{R}^{2}\end{array}$} \\
\hline $\begin{array}{c}\text { Dependent } \\
\text { variable(s) for } \\
y n \& y c\end{array}$ & $\begin{array}{c}\text { Method } \\
x \\
\left(\text { for } g m_{x}\right)^{* 1}\end{array}$ & $\begin{array}{l}\text { Somatic } \\
\text { growth } \\
g m_{\mathrm{x}}\end{array}$ & $\begin{array}{c}\text { Nauplii } \\
\text { production } \\
y n_{x}\end{array}$ & $\begin{array}{c}\text { Cysts } \\
\text { production } \\
y c_{\mathrm{x}}\end{array}$ & & \\
\hline \multicolumn{7}{|c|}{ Food used/growth (estimate \pm standard error, $d f$ ) } \\
\hline \multicolumn{7}{|c|}{ Estimated mainly by the regression } \\
\hline$R m_{\mathrm{i}} \& R m_{\mathrm{r}}$ & ir & $1.122 \pm 0.303,32$ & $4.438 \pm 0.055,32$ & $2.292 \pm 0.030,32$ & 1.936 & 0.835 \\
\hline$R m_{r}$ & rr & $1.833 \pm 0.307,32$ & $4.352 \pm 0.055,32$ & $2.237 \pm 0.030,32$ & 1.945 & 0.828 \\
\hline$R y_{\mathrm{r} 1}$ & rl & $1.520 \pm 0.488,16$ & $4.386 \pm 0.047,33$ & $2.261 \pm 0.025,33$ & 1.940 & 0.829 \\
\hline$R y_{\mathrm{r} 2}$ & r2 & $2.095 \pm 0.452,16$ & $4.313 \pm 0.047,33$ & $2.217 \pm 0.025,33$ & 1.945 & 0.826 \\
\hline$R y_{r 3}$ & г3(3) & $g m_{3}, 14$ & $4.154 \pm 0.132,16$ & $2.092 \pm 0.068,16$ & 1.986 & 0.645 \\
\hline$R y_{\mathrm{ri1}}$ & ril (i1) & $1.292 \pm 0.185,16$ & $4.416 \pm 0.046,33$ & $2.279 \pm 0.024,33$ & 1.938 & 0.836 \\
\hline$R y_{\mathrm{ri} 2}$ & ri2 (i2) & $1.489 \pm 0.187,16$ & $4.390 \pm 0.046,33$ & $2.264 \pm 0.024,33$ & 1.939 & 0.835 \\
\hline Mean (except r3) & & $1.558 \pm 0.321$ & $4.383 \pm 0.049$ & $2.258 \pm 0.026$ & 1.941 & 0.832 \\
\hline Mean (all) & & $1.558 \pm 0.321$ & $4.350 \pm 0.056$ & $2.235 \pm 0.030$ & 1.947 & 0.805 \\
\hline$R y_{\mathrm{riza}}^{* 2}$ & ri2a (i2a) & $1.489 \pm 0.187,16$ & $4.209 \pm 0.116,33$ & $2.272 \pm 0.061,33$ & 1.853 & 0.742 \\
\hline \multicolumn{7}{|c|}{$y n_{\mathrm{x}}$ and $y c_{\mathrm{x}}$ estimated independently } \\
\hline$R y_{\mathrm{r} 1}$ & $\mathrm{rl}$ & 1.520 & 5.460 & 2.274 & 2.400 & \\
\hline$R y_{\mathrm{r} 2}$ & r2 & 2.095 & 5.370 & 2.226 & 2.413 & \\
\hline$R y_{\mathrm{r} 3}$ & $r 3(3)$ & $g m_{3}$ & 5.171 & 2.090 & 2.474 & \\
\hline$R y_{\mathrm{ri} 1}$ & ril (il) & 1.292 & 5.495 & 2.294 & 2.396 & \\
\hline$R y_{\mathrm{ri} 2}$ & ri2 (i2) & 1.489 & 5.464 & 2.277 & 2.400 & \\
\hline Mean (except r3) & & 1.599 & 5.446 & 2.267 & 2.403 & \\
\hline Mean (all) & & 1.599 & 5.392 & 2.232 & 2.416 & \\
\hline$R y_{x i 2 a}^{* 2}$ & ri2a (i2a) & 1.489 & 5.000 & 2.277 & 2.195 & \\
\hline
\end{tabular}

Refer Appendix for variables.

*1: When $x^{\prime} s$ for $g m$ and for $y c$ and $y n$ are different, both are shown as stated.

*2: After correction of the assimilation efficiency in Method ri2. For further explanation see the text (Discussion, p. 501R). 


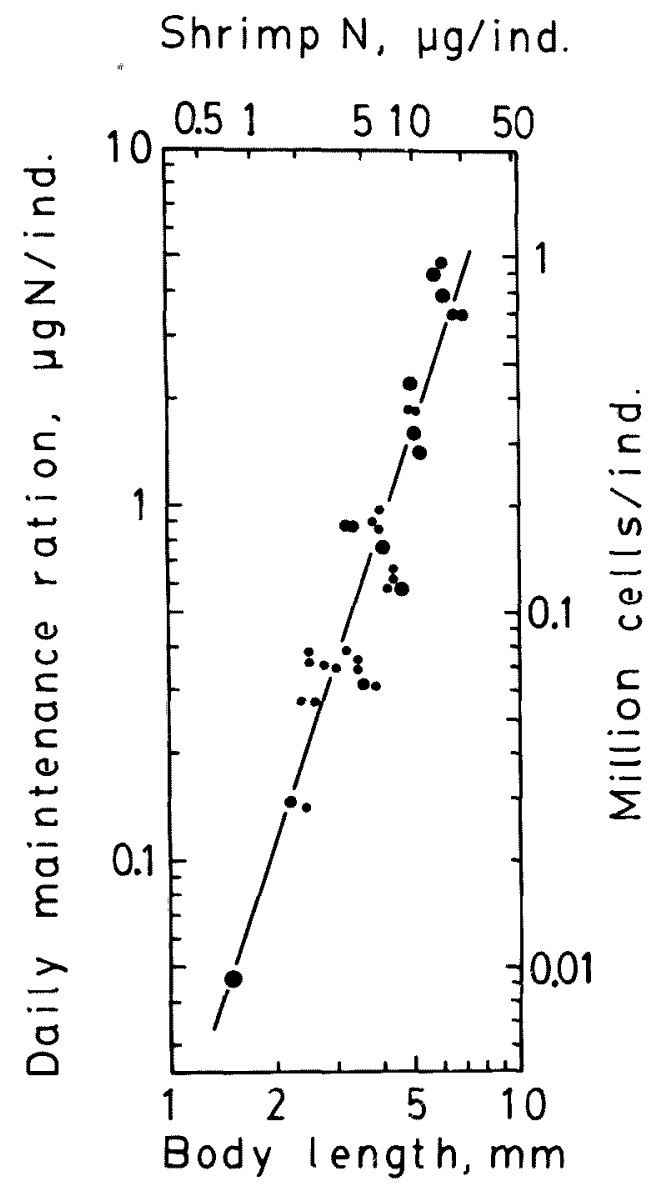

Fig. 4. Daily maintenance ration of Artemia franciscana related to body size.

The area of circles is proportional to the number of data $(\leq 4)$. The solid line is the regression line.

for the cyst-producing groups. Groups 26-35, on the other hand, produced only nauplii. Similarly, $y n_{\mathrm{x}}(\simeq 5.4)$ was obtained from the nauplius-producing groups. These are shown in the lower part of Table 4.

Although the total offspring produced by a pair differed among the groups, the mean standard deviation of brood size in each pair was $27.8 \%$.

\section{Ratio of Reproductive Products to Total Growth}

The growth is composed of somatic growth $G$ and reproductive growth $(Y n+Y c) / 2$. The ratio $(Y)$ of reproductive products to total growth was affected by the body size (age) and feeding rate: The simple correlation coefficients of $(Y c+Y n) /(2 \cdot G+Y c+Y n) v s$. body length and $v s . d n / m_{\mathrm{r}}$ (the ratio of daily ration to daily maintenance ration) were 0.892 and 0.672 (for groups 1-35), respectively (Fig. 6).

When the ratio of all spawn to total growth is expressed as $Y$, body length as $L \mathrm{~mm}$ and the ratio $d n / m_{\mathrm{r}}$ as $X$, the regression equation were obtained as follows:

$$
\begin{aligned}
& Y=1.197-0.348(L-5.73)^{-0.2045} \cdot(X-1)^{0.0059}, \\
& L>5.73 \text { and } X>1 \\
& Y=0, \quad L \leq 5.73 \text { or } X \leq 1
\end{aligned}
$$

Assuming that the regression line passes through the point

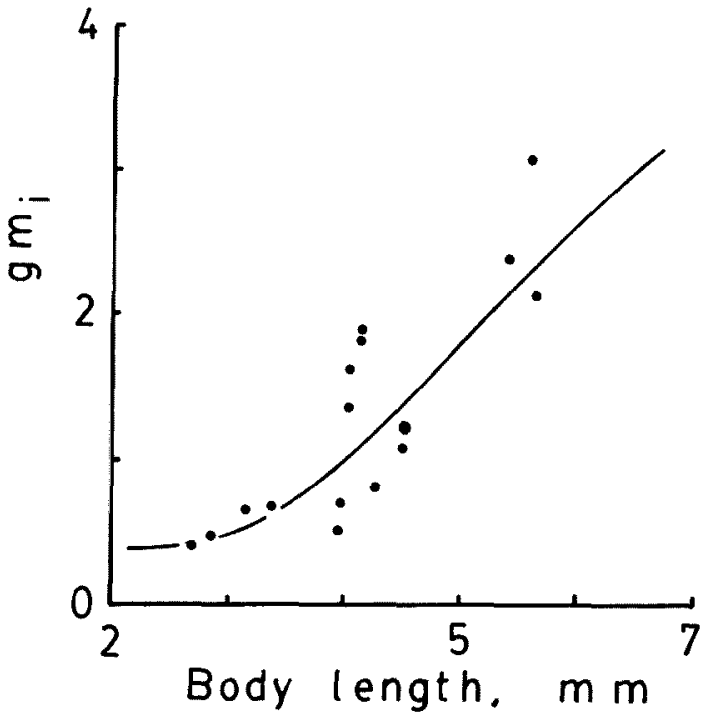

Fig. 5. Somatic food conversion factor $\left(g m_{\mathrm{i}}\right)$ related to body length The area of circles is proportional to the number of data $(\leq 2)$. The line is the regression line, from which $\mathrm{gm}_{3}$ was estimated.

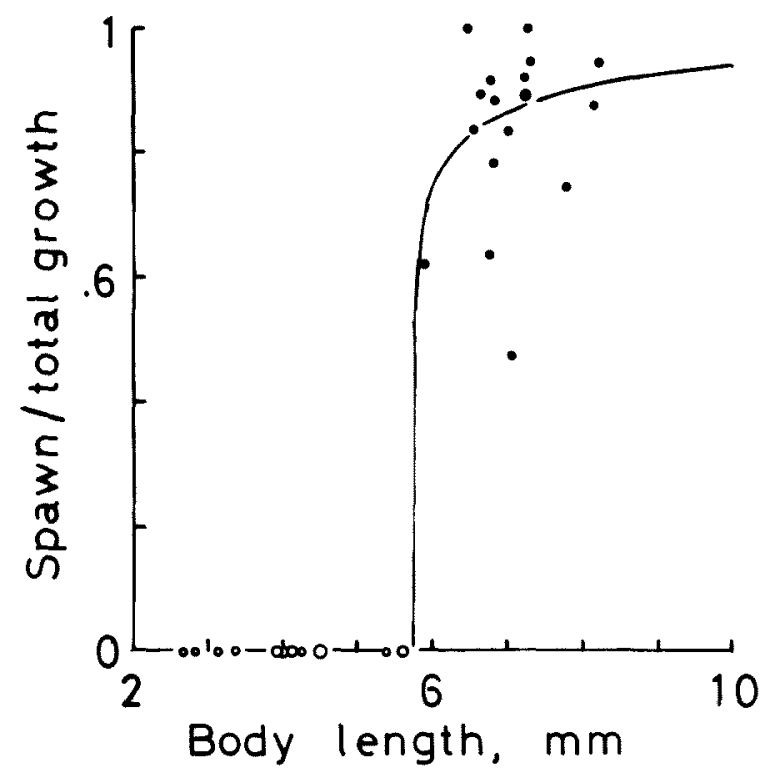

Fig. 6. Ratio of reproductive growth to total growth related to body length.

The reproductive growth $(Y n+Y c)$ is the sum of spawns per pair during the experiment and the total growth is the sum of somatic $(G)$ and reproductive growth. The area of circles is proportional to the number of data $(\leq 2)$. Solid circles were used to estimate the regression equation (13), open circles were not used. The regression line is for a feeding rate of three maintenance rations $(X=3.0)$, which was the mean.

( $L=5.73, X=1$ ), then the coefficient of determination due to regression was 0.954 for all the original data (groups $1-35$ ) and 0.250 for the spawners data (groups 18-35). However, the coefficient was 0.668 for the log-transformed spawners.

This shows that a well-fed large shrimp uses more food for reproduction than somatic growth. However, the effect of the daily ration is small. 


\section{Discussion}

The critical food concentration is known to be $41-233 \times$ $10^{3}$ cells $/ \mathrm{m} /$ for the used shrimp. ${ }^{3)}$ Unfortunately, however, the initial food concentration exceeded this sometimes in the rearing experiment. The period of maximum ingestion ranged from 0 to $5 \mathrm{~h}$ in most cases, but that in group 27 was $13 \mathrm{~h}$ sometimes. This maximum feeding might introduce an error in the estimation of ration, because the used formula (3) is only applicable when the food concentration is less than the critical value. Fortunately, however, it introduced a positive error of less than $5 \%,{ }^{7}$ because the daily specific growth rate of food was 0.665 per day and was less than the standard error of food concentration estimation. Furthermore, the difference between the actual and the estimated filtering rates is not considered to introduce a large error as shown in Table 1.

Although it appears confusing, the models of the energy budget and the mass budget are slightly different. ${ }^{11)}$ Even among mass budget models, there are some differences according to the products, processes and methods involved, which makes it difficult to compare data directly. Therefore, it is important to define the used model accurately. Our main concerns were the maintenance ration, somatic growth and reproduction. The maintenance ration was assumed to vary with the body size under a fixed condition. The other food conversion factors were assumed to be constant irrespective of body size at first, though some models for cattle and sheep assume that the somatic conversion factor increases with body size. ${ }^{12)}$ However, it is very difficult to determine how much of the ration contributes to the somatic growth and how much contributes to the reproductive growth separately, because the reproductive organs start to develop earlier than the initial reproduction. This might influence the somatic conversion factor such that the factor increases with body size ( $c f$. Fig. 5). Additionally, the maximum size of groups 1-17 (non-spawners) was larger than the minimum size of spawners (groups 18-35). This implies that the biological minimum size may also change with age ( $c f$. Fig, 6 and the last part of Discussion).

The gross conversion efficiency obtained here (16 and $24 \%$ in Tables 2 and 3 ) was similar to values already reported..$^{3.13-22)}$ Although the efficiency is easy to obtain, it is greatly affected by the feeding rate due to the subtraction of maintenance cost. However, the factors ( $g m, y c$, and $y n$ ) in our model are not affected under the definition used.

The somatic food conversion factor $g m$ includes not only the materials which are composed of somatic mass increment but also the metabolic cost for growth, such as the energy loss and excretion over the maintenance. The cost of molting is also included: the shed shell, for example, is considered to be similar to excreta. The time delay between the mass and length growth was ignored here. However, this delay was so small that it did not introduce a large error in the calculation ( $c f$. Fig. 3). Two other factors ( $y n$ and $y c$ ) are similar, the materials for new-born and also the metabolic cost for reproduction including those made by males.

The specific daily maintenance ration increased with the size of shrimp as shown in Fig. 7, ranging from 5 to $35 \% /$ day. The specific nitrogen excretion rate was reported to range from 6 to $22 \%$ day for the shrimp of $4-10 \mathrm{~mm}$

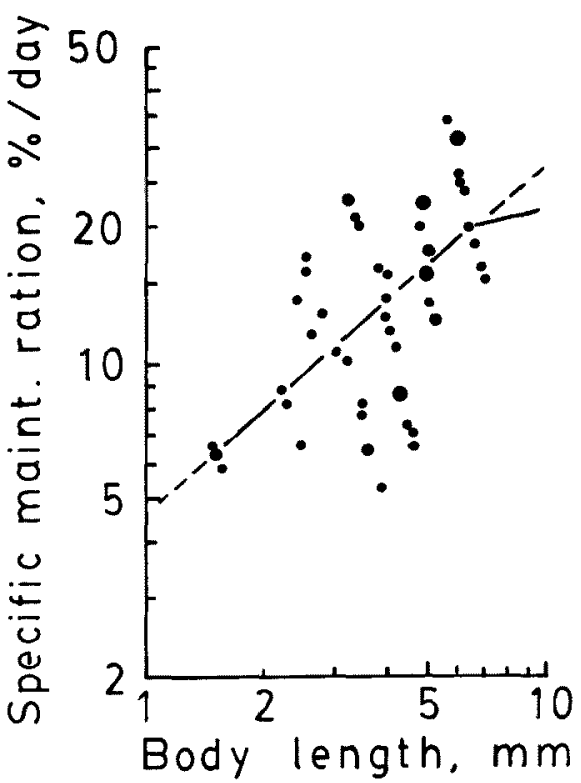

Fig. 7. Specific daily maintenance ration related to body size Specific maintenance ration ( $\mathrm{smr}$, specific daily maintenance ration, $\% /$ day) is plotted against body length $L$. The area of circles is proportional to the number of data $(\leq 3)$. The broken line is the regression line directly obtained from the data shown here: $s m r=$ $4.698 \cdot L^{0.767}$. The solid rectilinear line is obtained from the regression lines (Eqs. (1), (2), and (11)): $s m r=4.650 \cdot L^{0.776}(L \leq 6.3)$ and $s m r=12.73 \cdot L^{0.227}(L>6.3)$.

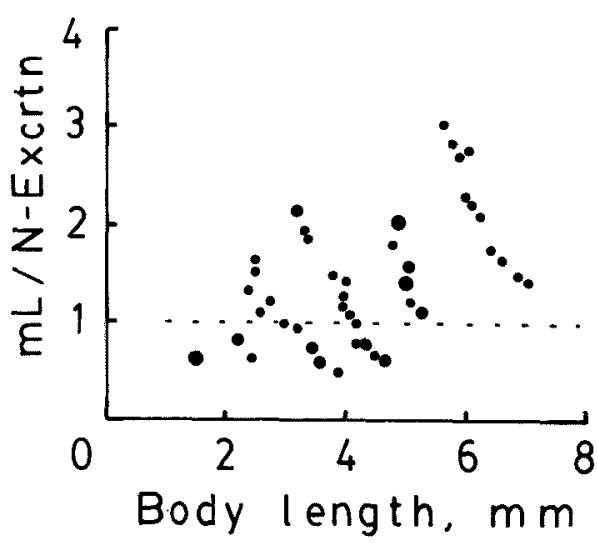

Fig. 8. Ratio of daily maintenance ration $(m L)$ to daily nitrogen excretion rate $(N-$ Excrtn) related to body length.

The area of circles is proportional to the number of data $(\leq 3)$ The broken line shows a ratio of unity.

length and decreased as the shrimp grew in contrast to the above rate. ${ }^{4)}$ The slopes of the log-log plot of the rates and body length were different $(p<0.001, t$-test). Although it is not clear at present, this might be caused from the definition itself. The daily maintenance ration does not include the metabolic cost for growth, but the observed excretion rate might include the cost. ${ }^{4}$ )

The ratio of daily maintenance ration to daily nitrogen excretion rate ${ }^{4)}$ is shown in Fig. 8. Although this ratio must have been larger than unity, it actually ranged $0.48-3.01$ for all the shrimps $(1.5-7.0 \mathrm{~mm})$ and $0.58-3.01$ for larger shrimps $(4.0-7.0 \mathrm{~mm})$ whose nitrogen excretion rate was obtained, ${ }^{4)}$ The mean for larger shrimps was $1.598 \pm 0.1153$ 
(SE, $N=35$ ). This can be used to estimate the real assimilation efficiency as $1 / 1.598(=62.6 \%)$, though it was assumed to be unity initially as shown in Materials and Methods. However, this estimate must be an underestimate because the rates were different in the character as described above. Even so, the estimate is similar to those reported on an amphipod, ${ }^{19)}$ isopod, ${ }^{19)}$ and copepods. ${ }^{18-21)}$

The somatic conversion factor was estimated directly by six methods as shown in Table 4 , and varied considerably. Additionally a seventh estimate $\mathrm{gm}_{3}$ was also obtained from the regression equation (12) in Fig. 5 as shown in Tables 2 and 3 . It is very difficult to select one of these factors because the coefficients of determination due to regression and the other factors $\left(y n_{\mathrm{x}}\right.$ and $\left.y c_{\mathrm{x}}\right)$ were very similar except the seventh estimate. The last estimate $g m_{3}$ was obtained by fitting a line to the independently-obtained $g m_{\mathrm{i}}$ without considering the theoretical nature of $g m_{\mathrm{i}}$. Although the correlation between $g m_{i}$ and body length $L$ was statistically significant $(p<0.01)$ and the coefficient of determination due to regression was 0.777 , one of the theoretical weak points in the regression is that $g m_{\mathrm{i}}$ approaches $0.384(<1)$ when the shrimp is small. This means that the sum of newly-formed tissue and the metabolic cost is less than the material (nitrogen) in the food. Even if we neglect food sources other than chlamydomonad, the neglected food would further reduce the estimate of daily maintenance ration because exactly the same rearing medium was used to estimate the daily maintenance ration and the somatic food conversion factor $g m_{\mathrm{j}}$. This asymptote $(<1)$ is reasonable when the bodily nitrogen estimated from the body length is overestimated and/or when the nitrogen content in chlamydomonad is underestimated. However, the data of bodily nitrogen of the shrimp $(2-4 \mathrm{~mm})$ were on or above the regression line, ${ }^{4)}$ and the nitrogen content in the phytoplankton ranged from 4.0 to $5.9 \mathrm{pg}$ per cell. ${ }^{31}$ It is therefore difficult to consider overestimation of bodily nitrogen and underestimation of algal nitrogen to give the asymptote. Even though the somatic conversion factor increased with body size as in cattle and sheep, ${ }^{12)}$ the observed conversion factor $g m_{\mathrm{i}}$ less than unity is considered here to be data with a mean larger than unity. So the seventh set of data is ignored here.

Of the remaining six sets of data, the standard errors of $g m_{\mathrm{i} 1}$ and $g m_{\mathrm{i} 2}$ were similar to each other and significantly smaller than the others $\left(p<0.05, F\right.$-test). The ratio $g m_{\mathrm{i} 2}$ is the ratio of mean ration over maintenance to mean somatic growth, while $g m_{i 1}$ is a simple mean of the ratio $\left(g m_{\mathrm{i}}\right)$ for each group. Although the standard errors were almost same, $g m_{\mathrm{i} 2}$ is adopted here as the better estimate because it is the weighted mean of $g m_{\mathrm{i}}$. The somatic food conversion efficiency is estimated to be $1 / 1.489(=67.2 \%)$. This is slightly larger than those of a copepod Acartia tonsa (44\% ingested C), ${ }^{22)}$ chub mackerel (18-30\% wet weight base),${ }^{23)}$ plaice, sole and perch (25-47\% ingested $\mathrm{N}$ and $28-49 \%$ assimilated $\mathrm{N}),{ }^{24)}$ but slightly smaller than that of carp $(74 \%$ assimilated energy, $92 \%$ assimilated $N){ }^{24,25)}$

The above selection of the estimation method automatically chooses the reproductive conversion factors $y n_{\mathrm{ri} 2}$ and $y c_{\mathrm{ri} 2} ; 4.390(22.8 \%$ in efficiency) and $2.264(44.2 \%$ in efficiency), respectively. These are significantly larger than $g m_{\mathbf{i} 2}$ (somatic conversion factor, $p<0.05, t$-test). Since the factors shown in the lower part of Table 4 were obtained by ignoring the data of the groups which produced both nauplii and cysts, they are not adopted here, but are fairly similar to the upper data.

Some shrimps ( $\# 27,28$, and 31 ) shown in Table 3 fed so well that the daily ration to bodily nitrogen was larger than unity. This fact might reduce the assimilation efficiency to 68,94 , and $80 \%$ respectively, according to Fig. 2 in our another paper. ${ }^{26)}$ When this correction was applied to the data, $y n_{\mathrm{i} 2}$ and $y c_{\mathrm{i} 2}$ changed to $4.209\left(=y n_{\mathrm{i} 2 \mathrm{a}}\right)$ and 2.272 $\left(=y c_{\mathrm{i} 2 \mathrm{a}}\right)$ as shown in Table 4 . However, since the coefficient of determination was reduced by $10 \%$, we ignored the correction here.

The egg production efficiency of a copepod Acartia tonsa ( $39 \%$ ingested $\mathrm{C}$ and $84 \%$ assimilated $C)^{20)}$ was similar to the $y c_{\mathrm{i} 2}$ obtained here. However, even the data on laying hens did not reveal the metabolic cost for egg production. ${ }^{12,27)}$ Although the nitrogen content per nauplius was smaller than that per cyst including shell, ${ }^{5)}$ the food needed to produce a nauplius was larger than the other. It is very difficult to understand the cause of this. After fertilization, the embryo which will become a cyst develops to the later stage of gastrula within the shell in the ovisac. ${ }^{28)}$ The other develops to a nauplius within the thin membranes in the ovisac. If the latter stays in the ovisac and is nourished by the mother even after fertilization, or if the ovoviviparous nauplius has more nitrogen than the oviparous embryo (since no data are available on the nitrogen content in the ovoviviparous new-born nauplius), then this explains the difference between the factors.

As is natural, somatic growth leads to reproductive growth. The smallest spawner was $5.82 \mathrm{~mm}$ in length (cf. Table 3) and the largest mean length of non-spawner (cf. Table 2) was $5.645 \mathrm{~mm}$. Hence, the body length of $5.73 \mathrm{~mm}$ was employed as the minimum size of reproductive shrimp in the regression calculation. However, this is an arbitrary value, because the largest non-spawner was $6.23 \mathrm{~mm}$ as shown in Table 2 , and $8.62 \mathrm{~mm}$ in the experiment. ${ }^{6)}$ The biological minimum may therefore also depend on age However, at the body length around this there are many kinds of deflection in the allometric growth, namely $3.6 \mathrm{~mm}$ in the maximal ingestion rate, ${ }^{3)} 4.0 \mathrm{~mm}$ in segmentation, ${ }^{29}$ ) $5.0 \mathrm{~mm}$ in gut content volume, ${ }^{1)} 5.8 \mathrm{~mm}$ in dry body weight ${ }^{4}$ and $6.3 \mathrm{~mm}$ in nitrogen content. ${ }^{4)}$ The similar intercept in feeding rate normalized to the daily maintenance ration was 1.00 (Fig. 6), because below this feeding rate the shrimp hardly maintained their own body and above this level the largest shrimp might only use the food for reproduction but not for somatic growth.

These data help us to construct a feeding program for the shrimp based on the assimilation efficiency and other data.

\section{References}

1) Y. Nimura: Shortest gut passage time and gut content volume of Artemia franciscana. Nippon Suisan Gakkaishi, 55, 2209 (1989).

2) D. A. Bengtson, P. Léger, and P. Sorgeloos: Use of Artemia as a food source for aquaculture, in "Artemia Biology" (ed. by R. A. Browne, P. Sorgeloos, and C. N. A. Trotman), CRC Press, Boca Raton, Fl., 1991, pp. 255-285.

3) Y. Nimura: Retarded growth of Artemia salina by overfeeding. Nippon Suisan Gakkaishi, 46, 681-687 (1980).

4) Y. Nimura and Md. I. Miah: Nitrogen excretion by Artemia 
franciscana. Nippon Suisan Gakkaishi, 57, 837-844 (1991).

5) J. Dutrieu: Observations biochimiques et physiologiques sur le developpement d'Artemia salina. Archs. Zool. exp. gén., 99, 1-133 (1960)

6) Y. Nimura: Effects of different concentrations of Chlamydomonas on the grazing and growth of Artemia with reference to the salinity of its living medium. Nippon Suisan Gakkaishi, 29, 424433 (1963) (in Japanese).

7) Y. Nimura: A new filter feeding model incorporating the critical food concentration. Nippon Suisan Gakkaishi, 46, 787-795 (1980).

8) Y. Tanaka, T. Tarumi, and K. Wakimoto: Multivariate Analysis, in "Handbook for Statistical Analysis by Personal Computer," Vol. 2 , Kyoritsu Shuppansha, Tokyo, 1984, pp. 1-15 (in Japanese).

9) K. Kawabata: Multiple regression, in "Handbook of Applied Statistics" (ed. by T. Okuno et al.), Yokendo, Tokyo, 1978, pp. 120-149 (in Japanese)

10) E. Kuno: Research Methods for Animal Population Dynamics, Vol. 1, Kyoritsu, Shuppansha, Tokyo, 1986, p. 8 (in Japanese).

11) H. Kawanabe and K. Onodera: Turnover of materials and energy, in "Research Methods of the Biological Production in Freshwater" (ed. by S. Mori et al.), Kodansha, Tokyo, 1969, pp. 352-356 (in Japanese).

12) H. Morimoto: Nutrition of Domestic Animals, Yokendo, Tokyo, 1985, pp. 332-333 (in Japanese)

13) L. M. Suschenya: Quantitative data on nutrition and energy balance in Artemia salina (L.). Dokl. Akad. Nauk SSSR, 143, 1205-1207 (1962) (in Russian).

14) M. R. Reeve: Growth efficiency of Artemia under laboratory conditions. Biol. Bull, 125, 133-145 (1963)

15) N. N. Khmeleva: Transformation of energy in Artemia salina (L.) Dokl. Akad. Nauk SSSR, 175, 934937 (1967) (in Russian).

16) N. N. Khmeleva: The expenditure of energy on respiration, growth and reproduction in Artemia salina (L.). Biologia Morya, 15, 71-98 (1968) (in Russian)

17) A. Gibor: Conversion of phytoplankton to zooplankton. Nature, 179, 1304 (1957).

18) P. Calow: Conversion efficiencies in heterotrophic organisms. Biol. Rev., 52, 385-409 (1977).

19) L. M. Suschenya: Food rations, metabolism and growth of crustaceans, in "Marine Food Chain" (ed. by J. H. Steele), Oliver and Boyd, Edinburgh, 1970, pp. 127-141

20) T. Kiorboe, F. Mahlenberg, and K. Hamburger: Bioenergetics of the planktonic copepod Acartia tonsa: relation between feeding, egg production and respiration, and composition of specific dynamic action. Mar. Ecol-Prog. Ser, 26, 85-97 (1985).

21) R. Conover: Transformation of organic matter, in "Marine Ecology" (ed. by O. Kinne), Vol. 4, Wiley, Chichester, 1978, pp. 221-499.

22) U. Berggreen, B. Hansen, and T. Kiørboe: Food size spectra, ingestion and growth of the copepod Acartia tonsa during development: implications for determination of copepod production. Mar. Biol., 99, 341-352 (1988).

23) M. Takahashi and M. Hatanaka: Experimental studies on the utilization of food by young mackerel Pneumaphorus japonicus (Houttuyn). Nippon Suisan Gakkaishi, 24, 449-455 (1958).

24) L. Birkett: The nitrogen balance in plaice, sole and perch. $J$. expl. Biol., 50, 375-386 (1969)

25) V. S. Ivlev: Balance of energy in carps. Zool. Zh., 18, 303-318 (1938) (cited from ref. 24).

26) Y. Nimura, K. Nanba, and Md. I. Miah: Apparent Assimilation Efficiency in Artemia related to the body size and ingestion rate Fisheries Sci, 60, 505-510 (1994)

27) R. Onodera, S. Hoshino, H. Itabashi, T. Hino, Y. Akiba, and S. Hasegawa: Nutriton of Domestic Animals, Kawashima, Tokyo, 1989, p. 215 (in Japanese)

28) R. Benesch: Zur Ontogenie und Morphologie von Artemia salina L. Zool. Jb. Anat., 86, 307-458 (1969).

29) P. B. Weisz: The space-time pattern of segment formation in Artemia salina. Biol. Bull., 91, 119-140 (1946).

\section{Appendix}

Variables list in alphabetical order

Variable Definition/Explanation

$A=a^{\prime} \cdot R$ assimilated ration, $\mu \mathrm{g} \mathrm{N} /$ shrimp

$a^{\prime} \quad$ apparent assimilation efficiency, normally assumed here to be unity ( $c f$. Discussion)

$C_{0} \quad$ initial food concentration in algal suspension, which was same both in the experimental and in the control without any grazer, thousand Chlamydomonas cells $/ \mathrm{m} l$

Cf correction factor for daily ration (Table 1)

$d$ daily ration, thousand Chlamydomonas cells/ shrimp/day

dl daily ration at the initial level, thousand Chlamydomonas cells/shrimp/day

d2 daily ration at the second level, thousand

Chlamydomonas cells/shrimp/day

$d n \quad$ daily ration, $\mu \mathrm{g} \mathrm{N} / \operatorname{shrimp} /$ day $(=0.005 \mathrm{~d})$

$f$ daily filtering rate, $\mathrm{ml} / \mathrm{shrimp} /$ day

$G$ somatic (or individual) growth in a period $T$ or $T a, \mu \mathrm{g} \mathrm{N} /$ shrimp

$\mathrm{gm}$ food conversion factor for somatic (or individual) growth (food/growth)

$g m_{3}=10^{\left(-16.266 / L^{2}+0.798\right)}+0.384 \mathrm{gm}$ estimated by the regression between $g m_{\mathrm{i}}$ and $L$ (Eq. (12))

$g m_{\mathrm{i}}=R m_{\mathrm{i}} / G \quad g m$ in each rearing experiment on a group $g m_{\mathrm{i} 1}=$ Mean $\left(g m_{\mathrm{i}}\right)=1.292 \quad$ simple mean of $g m_{\mathrm{i}}$ $g m_{\mathrm{i} 2}=\Sigma R m_{\mathrm{i}} / \Sigma G=1.489$ weighted mean of $g m_{\mathrm{i}}$ $g m_{\mathrm{ir}}=1.122 \mathrm{gm}$ obtained as a multiple regression coefficient by letting $G, Y c$, and $Y n$ be independent variables and $R m_{\mathrm{i}}$ for groups $1-17$ and $R m_{\mathrm{r}}$ for groups $18-35$ be dependent one

$g m_{\mathrm{r}}=R m_{\mathrm{r}} / G \quad g m$, using $m_{\mathrm{r}}$ to estimate $R m_{\mathrm{r}}$ $g m_{\mathrm{r} 1}=$ Mean $\left(g m_{\mathrm{r}}\right)=1.520 \quad$ simple mean of $g m_{\mathrm{r}}$ $g m_{\mathrm{r} 2}=\Sigma R m_{\mathrm{r}} / \Sigma G=2.095 \quad$ weighted mean of $g m_{\mathrm{r}}$ $g m_{\mathrm{rr}}=1.833 \mathrm{gm}$ obtained as a multiple regression coefficient by letting $G, Y c$, and $Y n$ be independent variables and $R m_{\mathrm{r}}$ be dependent one $g m_{\mathrm{x}} \quad g m$ estimated by the method of $\mathrm{x}(\mathrm{x}=3, \mathrm{i}, \mathrm{r}, \mathrm{i} 1$, i2, $\cdots$ )

$j \quad$ appropriate interger in calculation

$L \quad$ body length, $\mathrm{mm}$

L1 body length at the initial growth cessation, $\mathrm{mm}$

L2 body length at the second growth cessation, $\mathrm{mm}$

Lla body length at the first spawing in the experiment, $\mathrm{mm}$

$L 2 a$ body length at the last spawing in the experiment, mm

$M \quad$ total maintenance ration in a period $T$ or $T a$, assuming that $a^{\prime}$ is unity, $\mu \mathrm{g} \mathrm{N} / \mathrm{shrimp}$

$M_{\mathrm{j}} \quad M$ estimated by each rearing experiment on a group, $\mu \mathrm{g} \mathrm{N} /$ shrimp

$M_{\mathrm{r}} \quad M$ estimated by using the regression equation (Eq. (11)), $\mu \mathrm{g} \mathrm{N} /$ shrimp

$m L \quad$ observed daily maintenance ration of a $L \mathrm{~mm}$ long shrimp, $\mu \mathrm{g} \mathrm{N} / \mathrm{shrimp} /$ day unless stated

$m_{i} \quad$ daily maintenance ratio of a given size of shrimp estimated by $\log$-log interpolation in each rearing group, $\mu \mathrm{g} \mathrm{N} / \mathrm{shrimp} /$ day 
$m_{\mathrm{r}} \quad$ daily maintenance ration of a given size of shrimp estimated by $\log \log$ regression between $m_{\mathrm{i}}$ and $L$ (Eq. (11)), $\mu \mathrm{g} \mathrm{N} /$ shrimp/day unless stated number of reproductive cycles during which the material for a brood is accumulated

$p$ specific daily growth rate in chlamydomonad, $1 /$ day

$R \quad$ total ration in a period of $T$ or $T a, \mu \mathrm{g} \mathrm{N} /$ shrimp $\mathrm{R}^{2} \quad$ coefficient of determination due to regression

$R m_{\mathrm{i}}=A-M_{\mathrm{i}}$ surplus food over maintenance, $\mu \mathrm{gN} /$ shrimp

$R m_{\mathrm{r}}=A-M_{\mathrm{r}} \quad$ similar as above

$R y_{11}=R m_{\mathrm{i}}-g m_{\mathrm{i} 1} \cdot G$ surplus food for reproduction in a period $T a, \mu \mathrm{g} \mathrm{N} /$ shimp

$R y_{\mathrm{i} 2}=R m_{\mathrm{j}}-g m_{\mathrm{i} 2} \cdot G \quad$ similar as above

$R y_{\mathrm{i} 3}=R m_{\mathrm{i}}-g m_{3} \cdot G \quad$ similar as above

$R y_{\mathrm{r} 1}=R m_{\mathrm{r}}-g m_{\mathrm{r} 1} \cdot G \quad$ similar as above

$R y_{\mathrm{r} 2}=R m_{\mathrm{r}}-g m_{\mathrm{r} 2} \cdot G \quad$ similar as above

$R y_{\mathrm{r} 3}=R m_{\mathrm{r}}-g m_{3} \cdot G \quad$ similar as above

$R y_{\mathrm{r} 11}=R m_{\mathrm{r}}-g m_{\mathrm{i} 1} \cdot G \quad$ similar as above

$R y_{\mathrm{r} 2}=R m_{\mathrm{r}}-g m_{\mathrm{i} 2} \cdot G \quad$ similar as above

$R y_{\mathrm{r} i 2 \mathrm{a}}=R m_{\mathrm{r}}-g m_{\mathrm{i} 2} \cdot G \quad$ similar as above, assuming that $a^{\prime}$ in some groups was not unity (cf. p. 501R)

$R y_{\mathrm{x}} \quad$ surplus food for reproduction estimated by the method $\mathrm{x}$ as shown above, $\mu \mathrm{g} \mathrm{N} /$ shrimp

ry food for reproduction per brood per pair, used

\section{as a unit in Fig. 2}

$S l p i=\log (m L 2 / m L 1) / \log (L 2 / L 1) \quad$ slope for each rearing group

$s m r=m L /$ body $\mathrm{N} \quad$ specific daily maintenance ration, $\% /$ day

$T \quad$ period between growth cessations, day

$\mathrm{Ta}$ period between the first and last spawning in the reproductive experiment, day

single (or short-term) experimental feeding period $(\fallingdotseq 1$ day), day

$V \quad$ volume of experimental suspension, $\mathrm{m} l /$ shrimp

$X=d n / m_{\mathrm{r}} \quad$ daily ration relative to the maintenance ration

$X \quad$ suffix to indicate the method of estimation such as $3, \mathrm{i}, \mathrm{i} 1, \mathrm{i} 2, \mathrm{ir}, \mathrm{r}, \cdots$ they are attached to $g m_{\mathrm{x}}$, $R y_{\mathrm{x}}, y c_{\mathrm{x}}$, and $y n_{\mathrm{x}}$

$Y=(Y c+Y n) /(2 \cdot G+Y c+Y n) \quad$ reproductive component in total growth, spawn/total growth

$Y_{C} \quad$ cysts production in a given period of $T a, \mu \mathrm{g} \mathrm{N} /$ pair

yc food conversion factor for cysts production (food cyst)

$y c_{\mathrm{x}} \quad y c$ estimated by the method $\mathrm{x}(\mathrm{x}=3, \mathrm{i}, \mathrm{r}, \mathrm{i} 1, \mathrm{i} 2, \cdots)$

$Y n$ nauplii production in a given period of $T a$, $\mu \mathrm{g} \mathrm{N} /$ pair

$y n$ food conversion factor for nauplii production (food/nauplius)

$y n_{x} \quad y n$ estimated by the method $\mathrm{x}(\mathrm{x}=3, \mathrm{i}, \mathrm{r}, \mathrm{i} 1, \mathrm{i} 2, \cdots)$ 